\title{
Apego y bienestar en mujeres en proceso de tratamiento del cáncer de mama
}

\author{
Yolanda Alonso ${ }^{1}$, Yolanda Fontanil ${ }^{2}$ y Esteban Ezama ${ }^{3}$ \\ 1 Departamento de Psicología, Universidad de Almería (España) \\ 2 Departamento de Psicologia, Universidad de Oviedo (España) \\ 3 Centro de Investigaciones Comunicacionales, Oviedo (España)
}

\begin{abstract}
Resumen: Este estudio explora la contribución del vínculo de apego al bienestar de las pacientes en tratamiento por cáncer de mama. Su objetivo es aportar conocimientos sobre cómo favorecer el afrontamiento del cáncer y de las dificultades que suelen concurrir con él. Un total de 58 mujeres que iban a comenzar un tratamiento de radioterapia por cáncer de mama informaron a través de cuestionarios sobre: síntomas y disfunciones relacionados con la enfermedad y el tratamiento, afectos positivos y negativos, bienestar emocional, contrariedades diarias y vínculo de apego. El análisis de regresión revela que el componente Temor al rechazo y al abandono del apego, la presencia de contrariedades - principalmente las económicas- y la presencia de síntomas físicos predicen el grado de bienestar. Los otros dos componentes del apego (Deseo de cercanía y Preferencia por la independencia) no presentan ninguna relación significativa. Contrariamente a lo esperado, la merma de capacidad funcional no predice una disminución del bienestar. Se discute sobre el peso diferencial de las variables médicas y psicosociales en la calidad de vida relacionada con la salud y se concluye la importancia de las intervenciones encaminadas a mejorar el estado emocional durante los tratamientos del cáncer a través de la mejora de las relaciones cercanas. Palabras clave: Apego adulto; Calidad de vida relacionada con la salud; Cáncer de mama; Contrariedades diarias; Temor al rechazo y al abandono.
\end{abstract}

\section{Introducción}

El interés por la comprensión de los fenómenos relacionados con la salud y la enfermedad desde el prisma del vínculo de apego es muy reciente, y los estudios publicados hasta ahora ofrecen datos muy significativos. El estilo de apego seguro se ha relacionado con menos conductas de riesgo para la salud, una mejor adherencia a los tratamientos, una mejor aceptación y adaptación al proceso de la enfermedad, y una más eficaz búsqueda de cuidados (Huntsinger y Luecken, 2004; Maunder y Hunter, 2008; McWilliams y Bailey, 2010). En el caso del cáncer, la mayor parte de los estudios realizados hasta ahora se interesan por la influencia de los diferentes estilos de apego en el afrontamiento de la enfermedad, basándose en la idea de que el diagnóstico del cáncer activa las conductas de apego (Mikulincer y Shaver, 2007; Tan, Zimmerman y Rodin, 2005). También se ha hipotetizado que el apego puede intervenir en desarrollo del cáncer y contribuir a su curso, en tanto que la mayoría de los estresores que conocidamente influyen en el sistema inmune son al mismo tiempo activadores de las conductas de apego (Bowlby, 1988).

Si algo caracteriza al cáncer es la sensación de amenaza. Por eso, la calidad del vínculo establecido entre la persona enferma y sus cuidadores debe de ser particularmente relevante para su bienestar emocional. En esta línea, la investigación más reciente apunta a que el estilo de apego

* Dirección para correspondencia [Correspondence address]:

Yolanda Alonso, Departamento de Psicología, Universidad de Almería, 04120 Almería (España).E-mail: yalonso@ual.es
Title: Attachment and wellbeing in women undergoing breast cancer treatment.

Abstract: This study investigates the contribution of the attachment to the wellbeing of patients during breast cancer treatment with the aim to provide evidence on how to enhance coping with cancer and its related difficulties. The sample consisted of 58 women who were about to begin radiotherapy for breast cancer. They completed questionnaires about: symptoms and dysfunctions related to disease and treatment, positive and negative affect, emotional wellbeing, daily hassles, and adult attachment. A regression analysis revealed that the component Fear of rejection and abandonment of attachment, the presence of daily hassles - mainly economic-, and physical symptoms predict the degree of wellbeing. The other two components of attachment (Desire for closeness and Preference for independence) show no significant relationships, and contrary to expectations, the loss of functional capacity does not predict a decrease in wellbeing. We discuss the differential weight of medical and psychosocial variables on health-related quality of life. Results emphasize the importance of interventions aimed at improving emotional state through improved relationships during cancer treatments.

Key Words: Adult attachment; Health-related quality of life; Breast cancer; Daily hassles; Fear of rejection or abandonment.

seguro contribuye a una mejor adaptación a las dificultades que conlleva el cáncer (Cicero, Lo Coco, Gullo y Lo Verso, 2009; Hunter, Davis y Tunstall, 2006; Lo et al., 2010; Schmidt, Blank, Bellizzi y Park, 2012). Muy recientemente se ha puesto de relieve la importancia del estilo de apego y del ajuste de la pareja cuidadora en cáncer de colon (Hall, Armstrong, Cabral y Kim, 2012) y pulmón (Porter et al., 2012). Hasta el momento no se ha estudiado la forma en que variables relacionadas con el apego influyen en el bienestar emocional de las mujeres con cáncer de mama.

El planteamiento general de este estudio es explorar la contribución del vínculo de apego al bienestar durante el afrontamiento del cáncer y de las dificultades que suelen concurrir con él (dolor, capacidad funcional mermada, dificultades laborales o económicas, etc.), con el objetivo de aportar conocimientos sobre cómo favorecerlo. El cáncer conlleva dos tipos de cambios vitales. Por un lado pone a pacientes y allegados ante la perspectiva de la muerte. Por otro, las intervenciones médicas y los fuertes y largos tratamientos suponen una grave perturbación de la vida cotidiana. Desde la psicología del desarrollo, la vida puede definirse como una sucesión de tareas encaminadas a alcanzar ciertas metas (ya sean cotidianas o perentorias) y la construcción de las habilidades que se necesitan para ello (Havighurst, 1972). La persona enferma o convaleciente es un individuo en desarrollo obligado por la enfermedad a afrontar tareas nuevas, con una probabilidad aumentada de que diferentes metas entren en conflicto, y todo ello en una situación de recursos y habilidades mermadas o limitadas. Siguiendo este planteamiento, consideramos que las contrariedades diarias, entendidas como el estrés percibido 
relacionado con el desenvolvimiento cotidiano, son un medidor sensible de los cambios introducidos por el cáncer en la vida de las personas, y también los que de forma más directa pueden menoscabar el bienestar (Fillion, Kohn, Gagnon, van Wijka y Cunningham, 2001). Desde nuestro punto de vista, el estudio de las contrariedades diarias tiene la ventaja de que reflejan no solamente el esfuerzo personal por superar las dificultades cotidianas, sino el de las figuras cercanas, puesto que el éxito o el fracaso en la consecución de nuestras metas depende de los obstáculos o las facilidades que nos pongan los demás (Ezama, Alonso y Fontanil, 2010).

Con base a este planteamiento se diseñó un estudio predictivo transversal (Ato, López y Benavente, 2013) para explorar la relación existente entre las variables estudiadas. Como primera hipótesis, esperamos encontrar correlaciones significativas entre la apreciación personal de las pacientes sobre su estado de salud y su capacidad funcional, y el bienestar, entendido éste como la autovaloración del estado de ánimo. En segundo lugar, según nuestra visión evolutiva de la vida, esperamos encontrar también una relación significativa entre el bienestar y la presencia de contrariedades diarias y el bienestar y ciertas dimensiones del apego. Por último, nuestra intención es explorar cuál es el peso diferencial de las variables estudiadas para predecir el bienestar, y en todo caso esperamos que el apego sea una variable que prediga el bienestar, que al menos la suma total de las contrariedades también lo prediga, y que estas variables tengan un peso comparable al de las variables de salud.

\section{Método}

\section{Participantes}

En el estudio participaron un total de 58 mujeres, pacientes del Hospital Universitario Central de Asturias (HUCA), España, que habían sido diagnosticadas e intervenidas por un cáncer de mama en diferentes estadios y que iban a iniciar un tratamiento de radioterapia. La muestra se compuso con aquellas mujeres que contaban con un nivel educativo que les permitía usar los instrumentos de evaluación que fueron utilizados en la investigación. La edad media de las pacientes es de 52.63 años $(D T=10.7$; rango $29-78)$. El 72.5\% conviven con su pareja (casadas o no), el $12.7 \%$ están solteras, el $7.8 \%$ viudas, el $5.9 \%$ separadas/divorciadas y $1.7 \%$ tiene pareja sin convivir. En cuanto a su nivel de estudios, el $4 \%$ tiene un doctorado, el $40 \%$ son licenciadas universitarias, el $20 \%$ diplomadas, el $20 \%$ ha terminado la educación secundaria, el $12 \%$ la primaria y un $4 \%$ no tiene estudios. En cuanto a su situación médica, el 1.7\% no fue sometido a cirugía, el $75.8 \%$ había sufrido cirugía conservadora y el $22.4 \%$ mastectomía. El 40\% había recibido tratamiento de quimioterapia.

\section{Instrumentos}

Escala de Afectos Positivos y Negativos de SánchezCánovas (1994). Cuestionario de 20 ítems distribuidos en dos subescalas, Afectos Positivos (AP) y Afectos Negativos (AN). Cada una de ellas consistente en 10 adjetivos que expresan los estados de ánimo habituales (alegre, feliz, decidido...; deprimido, furioso, tenso...) en una escala de 5 puntos (de "muy poco o nada" a "mucho"). Las participantes debían contestar en qué grado habían experimentado esos estados de ánimo en el último mes. Se trata de la versión española del cuestionario PANAS (Positive and Negative Affect Schedule) de Watson, Clark y Tellegen (1998), cuya consistencia interna para la población española tiene valores de $\alpha$ de entre $.87 \mathrm{y}$ .91 (Sandín et al., 1999). En la muestra de este estudio, la consistencia interna fue de $\alpha=.89$ para la escala AP y de $\alpha=$ .93 para la escala AN.

Índice de Bienestar Psicológico (IBP), adaptación al español de Badía, Gutiérrez, Wiklund y Alonso (1996) del Psychological Well Being Index de Dupuy (1984). Consta de 22 ítems con respuesta tipo Likert de seis alternativas, que expresan el grado o intensidad de ocurrencia del enunciado del ítem desde que le diagnosticaron la enfermedad. Obtiene datos en seis dimensiones relacionadas con el bienestar: Ansiedad, Depresión, Estado de ánimo, Vitalidad, Autocontrol y Salud general. En el presente trabajo se utilizó únicamente la escala que mide el estado de ánimo (IBP-EA). El objeto de utilizar esta subescala es poder contar con una apreciación de bienestar para el periodo comprendido desde el diagnóstico evitando aplicar la Escala de Afectos Positivos y Negativos dos veces seguidas para periodos de tiempo parcialmente superpuestos. La consistencia interna de las respuestas de las participantes a este grupo de ítems es de $\alpha=.95$. Los estudios de validación de esta escala estimaron su grado de confiabilidad $\alpha=.94$ (Badía et al., 1996)

European Organization for Research and Treatment of Cancer Quality of Life Questionnaire (EORTC QLQ), en su adaptación española para cáncer de mama de Toledo, Barreto, Pascual y Ferrero (1993). Evalúa la vivencia de la enfermedad descrita por la propia paciente. Es un cuestionario de 53 ítems que combina preguntas con respuesta dicotómica y respuestas tipo Likert de 4 y 7 puntos. En el presente estudio se utilizaron dos de las ocho subescalas que ofrece el cuestionario: Nivel funcional $(\mathrm{NF})$ que evalúa el grado en que las pacientes se han visto capaces de realizar actividades de la vida cotidiana, relacionadas con el autocuidado, la movilidad o la actividad física; y Sintomas físicos (SIN), que evalúa el grado en que las pacientes perciben la presencia de síntomas relacionados con la enfermedad y el tratamiento, como dolor, falta de sueño, fatiga, alteraciones gastrointestinales, etc. La consistencia interna de las subescalas en la presente muestra fue $\alpha=.74$ y $\alpha=.87$ respectivamente. Toledo et al. (1993) encontraron para este instrumento valores de $\alpha$ entre .62 y .80 .

Escala de experiencias vitales recientes (SRLE) de Kohn y Macdonald (1992), en su versión española de Sandín, 
Chorot y Santed (1999). Es una valoración de estrés percibido. Consta de 51 ítems que enumeran experiencias o contrariedades frecuentes en la vida de las personas y evalúa el grado en que las pacientes están soportando problemas en sus vidas. Abarca seis áreas que corresponden a otras tantas subescalas: Dificultades sociales y culturales (conflictos con los amigos, decisiones sobre relaciones íntimas), Trabajo (insatisfacción laboral, conflictos con superiores), Presión del tiempo (exceso de tareas o de responsabilidades), Finanzas (tener cargas económicas), Aceptabilidad social (sentirse ignorado) y Victimización social (ser timado, estafado o maltratado). Las pacientes contestan estimando la intensidad con que han experimentado esas dificultades desde el diagnóstico de la enfermedad hasta el momento actual, según una escala tipo Likert, con un rango entre 1 y 4. En el presenta trabajo se han tenido en cuenta los problemas en conjunto (puntuación total), así como los problemas en cada una de las áreas. Los datos de consistencia interna fueron $\alpha$ $=.92$ para la puntuación total y valores de entre .56 y .78 para las subescalas. En estudios previos, esta escala ha obtenido valores de consistencia interna de .91 y .92 (Sandín et al., 1999).

Escala de Preferencias y Expectativas en las Relaciones Interpersonales Cercanas (EPERIC) de Fontanil, Ezama y Alonso (2013). Cuestionario de apego adulto de 22 ítems de respuesta tipo Likert de 5 puntos, que obtiene puntuaciones en tres subescalas que corresponden a tres componentes del apego: el Temor al rechazo y al abandono (TEMOR), el Deseo de cercanía (CERCA) y la Preferencia por la independencia (INDEP). Los valores de consistencia interna en nuestra muestra fueron de $\alpha=.72$ para la escala completa, $\mathrm{y}$ $\alpha=.81, \alpha=.70$ y $\alpha=.59$ para las tres subescalas respectivamente. En el trabajo de validación de la escala, los citados autores han encontrado valores de $\alpha$ de .80 para la escala completa y de entre .71 y .82 para las subescalas.

\section{Procedimiento}

Las participantes fueron mujeres que acudían al centro hospitalario para recibir tratamiento de radioterapia por cáncer de mama y a las que se les ofreció participar en un programa de apoyo psicológico. El proyecto fue evaluado y aprobado por el comité de ética del HUCA. Tras someterse a las evaluaciones preceptivas por el equipo médico, la psicoterapeuta encargada del programa llevaba a cabo una entrevista en la que eran informadas del trabajo psicoterapéutico que se iba a realizar y también del estudio y sus objetivos, al tiempo que se les solicitaba el consentimiento informado. La tasa de aceptación para participar en la investigación fue del 71.8\%. Solamente tres de las mujeres que tomaron parte en el programa de apoyo psicológico rehusaron proporcionar datos para el estudio. El resto no quiso o no pudo participar en el programa o faltó a las primeras citas sin que se pudiese reanudar el contacto. Las participantes rellenaron los cuestionarios en el centro hospitalario coincidiendo con las citas para las sesiones con la psicoterapeuta.

\section{Análisis de datos}

Se utilizaron en primer lugar correlaciones de Spearman para hallar las relaciones estadísticamente significativas entre las variables. A continuación se realizó un análisis de conglomerados de $\mathrm{K}$ medias partiendo de las puntuaciones en las variables criterio (escalas AP, AN y IBP-EA), y la muestra se dividió en dos grupos para cada subescala. El grupo "mejor" en AP y en IBP-EA es el de puntuaciones más altas y el grupo "peor" el de puntuaciones más bajas, y al contrario para la escala AN. De esta manera la variable criterio (bienestar) queda dicotomizada y operativizada por la pertenencia al grupo "mejor" o "peor" en cada subescala.

Seguidamente, con el objeto de determinar el valor predictivo sobre el bienestar de las contrariedades cotidianas, las dimensiones del apego y el estado de salud percibido, se realizaron análisis de regresión logística binaria (método "adelante condicional" para eliminar las variables redundantes). Como variables criterio se tomaron tanto la presencia de afectos positivos como la ausencia de afectos negativos, así como la puntuación global en la escala Estado de ánimo del IBP. Para reducir el número de variables en la ecuación, se utilizó la correlación de cada una de las variables predictoras con las escalas de bienestar, y se introdujeron en el cálculo de la ecuación de regresión solo aquellas variables cuya relación con la variable criterio era estadísticamente significativa al menos al 5\%. Para analizar los datos se utilizó el programa de tratamiento estadístico SPSS (versión 17.0 para Windows).

\section{Resultados}

La Tabla 1 muestra las correlaciones entre las escalas de bienestar emocional y las de autoevaluación de la salud, el estrés percibido y las dimensiones del apego. Tanto el afecto positivo como el negativo correlacionan de forma significativa con el nivel funcional y la severidad de los síntomas, así como con la mayoría de las subescalas del cuestionario de estrés. Solamente la variable AP no correlaciona con Presión del tiempo. En cuanto a las dimensiones del apego, la escala TEMOR correlaciona de forma significativa con las tres escalas de bienestar. Las otras dos dimensiones no obtienen ninguna correlación significativa. La correlación de las dos variables de salud entre sí (Nivel funcional y Síntomas físicos) es de .575 ( $p$ $<.0001)$ 
Tabla 1. Correlaciones de Spearman entre los afectos positivos y negativos y las variables relacionadas con estrés, salud y apego.

\begin{tabular}{|c|c|c|c|c|c|c|c|c|c|c|c|c|c|c|c|}
\hline & \multicolumn{2}{|c|}{ PANAS } & IBP & \multicolumn{2}{|c|}{ EORTC } & \multicolumn{7}{|c|}{ SRLE } & \multicolumn{3}{|c|}{ EPERIC } \\
\hline & $\mathrm{AP}$ & $\mathrm{AN}$ & EA & $\mathrm{NF}$ & SIN & (total) & $\mathrm{SOC}$ & TR & TIE & FIN & ACEP & VIC & TEMOR & CERCA & INDEP \\
\hline$\overline{\mathrm{AP}}$ & 1 & $-.767^{* *}$ & $.862^{* *}$ & $.510^{* *}$ & $-.505^{* *}$ & $-.484^{* *}$ & $-.314^{*}$ & $-.407^{* * *}$ & -.230 & $-.510^{* *}$ & $-.432^{* *}$ & $-.318^{*}$ & $-.466^{* *}$ & -.048 & -.145 \\
\hline $\mathrm{AN}$ & & 1 & $-.824^{* *}$ & $-.428^{* *}$ & $.494^{* *}$ & $.596^{* *}$ & $.405^{* *}$ & $.501^{* *}$ & $.379^{* *}$ & $.447^{* *}$ & $.365^{*}$ & $.432^{* *}$ & $.546^{* *}$ & -.068 & .238 \\
\hline IBP-EA & & & 1 & $.545^{* *}$ & $-.616^{* *}$ & $-.583^{* *}$ & $-.407^{* *}$ & $-.460^{* * *}$ & $-.339^{*}$ & $-.447^{* *}$ & $-.379^{* *}$ & $-.462^{* *}$ & $-.490^{* *}$ & -.100 & -.183 \\
\hline
\end{tabular}

Como se aprecia en la tabla, las variables que presentan una correlación más fuerte con los afectos positivos son las medidas del estado de salud y la subescala Finanzas del cuestionario de contrariedades, todas ellas con un coeficiente de correlación por encima de .5. A continuación vienen la suma total de contrariedades diarias y la dimensión TEMOR del apego. En cuanto a los afectos negativos, las relaciones más fuertes se establecen con el conjunto de contrariedades y con TEMOR, mientras que las medidas de salud, aunque también altamente significativas, se sitúan ligeramente por debajo de las anteriores. En cuanto a las experiencias diarias individualmente consideradas, la escala Trabajo es la de más alta correlación con AN. Las correlaciones de la medida global IBP-EA son muy similares a la de la escala AP, observándose las correlaciones más altas con SIN y NF y con la medida conjunta de contrariedades, y situándose inmediatamente por debajo la escala TEMOR.

El análisis de regresión permite saber qué variables son relevantes para predecir la pertenencia al grupo "mejor" o "peor" de cada subescala. Para la escala de afectos positivos, el grupo de puntuaciones más bajas (peor) se aglutina en torno al valor $2.07(n=27)$ y el grupo mejor en torno al 3.43 $(n=29)$. Para los afectos negativos, el grupo de puntuaciones más altas (peor) se agrupa en torno al valor $3.53(n=16)$ y el grupo mejor en torno al $1.63(n=40)$. En la escala global de estado de ánimo, el grupo peor está en torno al $2.98(n=$ 23) y mejor en torno al $4.67(n=35)$.

$\mathrm{El}$ análisis multivariante mediante regresión logística para la predicción de los afectos positivos dio lugar a los resultados que se muestra en la Tabla $2\left(\chi^{2}=21.461, g l=2, p<\right.$ $.001)$. En cuanto a la eficacia predictiva, los estadísticos indican que el modelo explica el $52.6 \%$ de la variabilidad de AP $\left(R^{2}\right.$ de Nagelkerke $\left.=.526\right)$. La tabla de clasificación muestra un $73.7 \%$ de clasificaciones correctas para el grupo peor y un $83.3 \%$ para el grupo mejor (punto de corte: .50). Como puede verse en la Tabla 2, solamente las subescalas Finanzas del cuestionario de contrariedades y la variable de salud Sintomas físicos entran en la ecuación.

Tabla 2. Modelo de regresión logística binaria para la predicción del afecto positivo.

\begin{tabular}{lrrrrrr}
\hline & \multicolumn{1}{c}{$B$} & \multicolumn{1}{c}{ E.T. } & \multicolumn{1}{c}{ Wald } & $g l$ & Sig. & $\operatorname{Exp}(B)$ \\
\hline Finanzas & -5.003 & 1.988 & 6.337 & 1 & .012 & .007 \\
Sintomas fisicos & -1.928 & .753 & 6.561 & 1 & .010 & .145 \\
Constante & 9.891 & 2.930 & 11.392 & 1 & .001 & 19746.089 \\
\hline
\end{tabular}

En lo que respecta a la predicción de los afectos negativos, el modelo, que se expone en la Tabla $3\left(\chi^{2}=34.039, \mathrm{gl}=\right.$ 4 , $p<0,0001)$, explica cerca del el $79 \%$ de la varianza $\left(R^{2}=\right.$
.788). Las clasificaciones correctas para el grupo peor son el $83.3 \%$ y el $93.5 \%$ para el grupo mejor, siendo el punto de corte .50 . En este caso, cuatro variables resultan predictoras de la variable criterio: la puntuación total del cuestionario de contrariedades, Sintomas físicos, Victimización social y Temor al rechazo y al abandono.

Tabla 3. Modelo de regresión logística binaria para la predicción del afecto negativo.

\begin{tabular}{lrrrrrr}
\hline & $B$ & E.T. & Wald & $g l$ & Sig. & $\operatorname{Exp}(B)$ \\
\hline Contrariedades (total) & -16.102 & 7.245 & 4.939 & 1 & .026 & .000 \\
Sintomas físicos & -4.446 & 1.912 & 5.404 & 1 & .020 & .012 \\
Victimización social & 4.166 & 2.012 & 4.290 & 1 & .038 & 64.475 \\
Temor al rechazo... & -3.940 & 1.723 & 5.229 & 1 & .022 & .019 \\
Constante & 38.095 & 16.222 & 5.515 & 1 & .019 & 3.502 \\
\hline
\end{tabular}

En la Tabla 4 se expone el modelo de regresión obtenido para la escala IBP-EA $\left(\chi^{2}=21.390, g l=2, p<0,0001\right)$. El modelo explica casi el $53 \%$ de la variabilidad $\left(R^{2}=.527\right)$. El modelo clasifica correctamente un $72.2 \%$ de casos para el grupo peor y un $88.0 \%$ para el grupo mejor (punto de corte: .50). Dos variables entran en la ecuación de regresión en este caso: Sintomas físicos y Temor al rechazo y al abandono.

Tabla 4. Modelo de regresión logística binaria para la predicción del estado de ánimo

\begin{tabular}{lrrrrrr}
\hline & \multicolumn{1}{c}{$B$} & \multicolumn{1}{c}{ E.T. } & \multicolumn{1}{c}{ Wald } & gl & Sig. & $\operatorname{Exp}(B)$ \\
\hline Sintomas físicos & -2.647 & .857 & 9.541 & 1 & .002 & .071 \\
Temor al rechazo ... & -1.298 & .557 & 5.427 & 1 & .020 & .273 \\
Constante & 7.777 & 2.169 & 12.855 & 1 & .000 & 2384.685 \\
\hline
\end{tabular}

\section{Discusión}

En este trabajo se han comprobado las relaciones entre algunas variables especialmente sensibles a los cambios vitales que acompañan al diagnóstico y tratamiento del cáncer de mama, y se ha explorado su valor predictivo sobre el bienestar, entendido éste como la presencia de afectos positivos y/o la ausencia de afectos negativos. Los resultados indican que el componente Temor al recharo y al abandono del vínculo de apego está fuertemente relacionado con el bienestar, como también lo está la presencia de contrariedades cotidianas y la presencia de síntomas relacionados con la enfermedad y el tratamiento.

Como era esperable, tanto la capacidad funcional de las pacientes como la gravedad de los síntomas que perciben correlacionan fuertemente con los medidores del estado de ánimo. Cuanto más alto es el nivel funcional y más baja la presencia de síntomas, más alto es el afecto positivo y más bajo el negativo. El mismo patrón se encuentra en la medida 
global del bienestar. Estos resultados concuerdan con los de Badr, Basen-Engquist, Taylor y de Moor (2006) que encuentran también relaciones significativas entre el estado de ánimo y la severidad de síntomas físicos en mujeres con cáncer de mama.

La presencia de contrariedades cotidianas ha demostrado también una fuerte relación con el bienestar. Consideradas de modo global, se puede afirmar que cuantas menos contrariedades, mayor bienestar. Considerándolas de forma diferenciada, los problemas relacionados con finanzas y laborales resultan ser los más relevantes.

Respecto a la relación entre estilo de apego y bienestar, también de acuerdo con lo esperado, la subescala Temor al rechazo y abandono parece tener un papel fundamental en el bienestar, tal que cuanto más temor tienen las pacientes a ser abandonadas o rechazadas en sus relaciones interpersonales, más informan de altas emociones negativas y de bajas emociones positivas. Es destacable que las subescalas Preferencia por la independencia y Deseo de cercanía no correlacionan con ninguna de las escalas de bienestar. Este resultado tiene una implicación clínica importante, ya que permite desestimar variables menos relevantes a la hora de ayudar a las personas que están en una situación de amenaza y cambio vital como el cáncer. Esto concuerda con otros estudios en los que el estilo de apego seguro (con un bajo componente de Temor al rechazo y al abandono) se ha relacionado con mejores puntuaciones en bienestar y otras variables relacionadas con el manejo de la enfermedad en pacientes con cáncer (Lo et al., 2010; Porter et al., 2012). No hemos encontrado ningún estudio sobre apego y cáncer en el que se analicen sus componentes separados en lugar de sus combinaciones (los estilos de apego).

Tomados los datos de forma global, la gravedad percibida de los síntomas resulta ser la variable más importante para predecir el bienestar, puesto que es la única que entra en las tres ecuaciones de regresión. Considerando el estado de ánimo desde la comunicación del diagnóstico, la dimensión Temor del apego la predice también. Considerando los afectos positivos y negativos durante el último mes y separadamente, se pueden constatar resultados más detallados. Los afectos positivos son sensibles sobre todo a los problemas relacionados con las finanzas. El afecto negativo es más complejo. La variable de más peso para predecirlo es la presencia de contrariedades en general, seguida por la severidad de los síntomas, los problemas relacionados con la victimización social (sentirse maltratado), y por el temor a ser rechazado o abandonado por las personas cercanas.

Contrariamente a lo esperado, la capacidad para realizar las funciones cotidianas no resulta predictora de ninguna de las escalas. Sentirse discapacitado parece irrelevante a efectos de bienestar emocional, mientras que la otra variable que recuerda directamente la enfermedad y su tratamiento, los Sintomas físicos, resulta crucial. Este llamativo resultado puede deberse a que la presencia de síntomas se interprete como una amenaza, y las limitaciones funcionales en cambio como una consecuencia pasajera y reversible de los tratamientos.
También hay que considerar que la movilidad reducida o las dificultades para realizar las funciones cotidianas no necesariamente cursan con malestar o dolor, potencialmente más perturbadores del bienestar, siendo éstos más característicos de los síntomas. En todo caso, estos resultados son llamativos por la elevada correlación que muestran ambas variables entre sí (véase también Snyder et al., 2008).

Consideración adicional merece el hecho de que el nivel funcional no forme parte de las ecuaciones de regresión a pesar de que sus correlaciones con las variables criterio son muy elevadas, especialmente con el afecto positivo. No cabe si no pensar que la afectación de la disfunción en el estado emocional está mediada por otras variables, probablemente por la presencia de, o el modo de enfrentarse a, problemas de la vida ajenos a la enfermedad (sin perjuicio de que estén relacionados con ella), como se puede colegir del hecho de que las contrariedades diarias sí predigan el bienestar, como factor además de primero orden. Otra posible variable mediadora sería la seguridad en las relaciones cercanas, por la misma razón.

El papel del Temor al rechazo y al abandono en la predicción de los afectos merece también especial reflexión, puesto que predice la ausencia de afectos negativos, pero no la presencia de afectos positivos. Esto sugiere que las personas con poco temor a ser rechazadas por sus seres queridos están mejor protegidas contra la aparición de emociones negativas (ira, depresión, ansiedad), pero que eso no garantiza que se experimenten sensaciones placenteras. Siguiendo otra línea de argumentación, las personas con más alto temor al rechazo y al abandono están más alerta, como es propio de los vínculos de apego de estilo preocupado o temeroso (Bartholomew y Horowitz, 1991; Fontanil et al., 2013) y por lo tanto más preocupadas o centradas en los aspectos negativos de la vida.

Numerosos estudios relacionan los eventos o experiencias estresantes con un mayor riesgo de contraer cáncer o con un peor pronóstico del mismo (Chida, Hamer, Wardle y Steptoe, 2008; Duijts, Zeegers y Borne, 2003) y también se dispone de datos de cómo el propio diagnóstico del cáncer actúa como estresante psicológico (Ando et al., 2011), pero no hemos encontrado en la literatura evidencias de cómo las experiencias estresantes afectan al proceso del tratamiento. Según nuestros resultados, la variable de más peso para predecir el afecto positivo es la ausencia de problemas económicos, mientras que el mejor predictor de los afectos negativos es la puntuación elevada en el conjunto del estrés cotidiano, aunque es particularmente relevante para sentirse mal que existan problemas de victimización social, tales como sentirse engañado o maltratado.

El bienestar de las pacientes con cáncer depende de múltiples variables, reducir su bienestar a un buen diagnóstico y tratamiento parece insuficiente. Desde un punto de vista clínico también es insuficiente considerar la ausencia de síntomas o un buen nivel funcional como descriptores del mismo. Nuestros resultados refuerzan la consideración de que las intervenciones oncológicas encaminadas a mejorar la calidad de vida no pueden olvidarse de resolver las dificultades in- 
terpersonales y emocionales de los pacientes (Cerezo, OrtizTallo y Cardenal, 2009; Hernández et al., 2012). Mejorar los lazos con los otros significativos mejorará la eficiencia de los tratamientos (Kim, Carver, Deci y Kasser, 2008; Bazzazian y Besharat, 2012; Tan et al., 2005). Según los resultados, la intervención más eficaz para mejorar el bienestar de las mujeres en proceso de terapia de cáncer de mama es aquella que preste ayuda para afrontar las contrariedades diarias. Y esto a su vez se consigue a través de la mejora de las relaciones cercanas. No solamente porque el impacto de los problemas cotidianos sea más bajo cuanto más alta sea la calidad de nuestras relaciones, sino también porque según los resultados de nuestras ecuaciones de regresión, es el Temor al rechazo y al abandono la variable que engloba el efecto de las contrariedades cotidianas. Cuando se afinan las medidas separando haberse sentido bien de no haberse sentido mal durante un plazo de tiempo más corto (último mes) podemos precisar qué contrariedades afectan más a las personas, pero cuando se hace una medida global del estado de ánimo desde la comunicación del diagnóstico desaparece la importancia de las contrariedades. Se puede decir entonces que el componente del apego Temor al rechazo y al abandono recoge toda la información necesaria para saber cómo predecir el bienestar afectivo.

El estilo de apego se mantiene relativamente estable a lo largo de la vida pero no es invariable. Las experiencias vitales, e incluso el mero transcurso de la vida pueden modificarlo (Grossmann, Grossmann y Waters, 2005; Mikulincer y Shaver, 2007), además de ser susceptible de mejora terapéutica (Gould, 2011; Wallin, 2007). Cuando una persona enferma, sus necesidades de atención y de apoyo no solo au-

\section{Referencias}

Ando, N., Iwamitsu, Y., Kuranami, M., Okazaki, S., Nakatani, Y., Yamamoto, K., Watanabe, M. y Miyaoka, H. (2011). Predictors of psychosocial distress after diagnosis in breast cancer patientes and patients with benign breast problems. Psychosomatics, 52(1), 56-64.

Ato, M., López, J.J. y Benavente, A. (2013). Un sistema de clasificación de los diseños de investigación en psicología. Anales de Psicología, 29(3), 1038-1059.

Badía, X., Gutiérrez, F., Wiklund, I. y Alonso, J. (1996). Validity and reliability of the Spanish Psychological General Well-Being Index. Quality of Life Research, 5, 101-108.

Badr, H., Basen-Engquist, K., Taylor, C.L.C. y de Moor, C. (2006). Mood states associated with transitory physical symptoms among breast and ovarian cancer survivors. Journal of Behavioral Medicine, 29, 461-475.

Baldwin, M.W., Keelan, J.P.R., Fehr, B., Enns, V. y Koh-Rangarajoo, E. (1996). Social-cognitive conceptualization of attachment working models: Availability and accessibility effects. Journal of Personality and Social Psychology, 71, 94-109.

Bartholomew, K. y Horowitz, L.M. (1991). Attachment styles among young adults: a test of a four-category model. Journal of Personality and Social Psychology, 61(2), 226-44.

Bazzazian, S. y Besharat, M.A. (2012). An explanatory model of adjustment to type I diabetes based on attachment, coping, and self-regulation theories. Psychology, Health \& Medicine, 17(1), 47-58.

Bowlby, J. (1988). A secure base: Parent-child attachment and healthy buman development. New York: Basic.

Cerezo, M.V., Ortiz-Tallo, M. y Cardenal, V. (2009). Expresión de emociones y bienestar en un grupo de mujeres con cáncer de mama: una intervención psicológica. Revista Latinoamericana de Psicología, 41(1), 131-140. mentan, sino que se activan otras específicas (Lo et al., 2009), que según los datos puede resumirse en incrementar la calidad de las relaciones cercanas a través sobre todo de una disminución del temor a ser rechazados o abandonados por las figuras de apego. Las intervenciones específicas que logren una mejora en el estado emocional conseguirán a su vez menor búsqueda de atención profesional, menor número de consultas y en general una mayor autonomía de la paciente con respecto a la estructura sanitaria que la atiende.

Una de las limitaciones de este estudio lo representa el pequeño tamaño de nuestra muestra. Sería recomendable contar con la participación de un mayor número de pacientes. Por otro lado, nuestro estudio utiliza una metodología transversal, mientras que hacer comparaciones en las diferentes fases del tratamiento, en las que las necesidades de las personas necesariamente cambian, aportaría información sin duda relevante. Si consideramos el estilo de apego no como un rasgo de personalidad que se construye desde la infancia y perdura en la edad adulta, sino como un estado (Baldwin, Keelan, Fehr, Enns y Koh-Rangarajoo, 1996; Collins y Read, 1994), es probable que las conductas de apego cambien en función de las amenazas percibidas. Si esto es así, las puntuaciones en los cuestionarios de apego deberían variar significativamente dependiendo del momento en que se lleve a cabo la evaluación. En estudios futuros nos proponemos explorar esta hipótesis relacionando evaluaciones realizadas en diferentes momentos del proceso de diagnóstico y tratamiento. Conocer el tipo de apoyo que las pacientes requieren en cada fase del afrontamiento del cáncer podrá ser útil para diseñar intervenciones más eficientes.

Chida, Y., Hamer, M., Wardle, J. y Steptoe, A. (2008). Do stress-related psychosocial factors contribute to cancer incidence and survival? Nature Clinical Practice Oncology, 5, 466-475.

Cicero, V., Lo Coco, G., Gullo, S. y Lo Verso, G. (2009). The role of attachment dimensions and perceived social support in predicting adjustment to cancer. Psycho-Oncology, 18(10), 1045-1052.

Collins, N.L. y Read, S.J. (1994). Cognitive representations of attachment: The structure and function of working models. En K. Bartholomew y D. Perlman (Eds.), Advances in personal relationships (Vol. 5, pp. 53-90). London: Kingsley.

Duijts, S.F.A., Zeegers, M.P.A. y Borne, B.V. (2003). The association between stressful life events and breast cancer risk: a meta-analysis. International Journal of Cancer, 107, 1023-1029.

Dupuy, H.J. (1984). The psychological general well-being (PGWB) index. En N.K. Wegner, M.E. Mattson y C.P. Fuberg (Eds.), Assessment of quality of life in clinical trials of cardiovascular therapies (pp. 170-183). New York: Le Jacq.

Ezama, E., Alonso, Y. y Fontanil, Y. (2010). Pacientes, síntomas, trastornos, organicidad y psicopatología. International Journal of Psychology and Psychological Therapy, 10(2), 293-314.

Fillion, L., Kohn, P., Gagnon, P., Van Wijka, M. y Cunningham A. (2001). The inventory of recent life experiences for cancer patients (IRLE-C): A decontaminated measure of cancer-based hassles. Psybology and Health, 16(4), 443-459.

Fontanil, Y. Ezama, E. y Alonso, Y. (2013). Validation of the scale of preferences and expectations in close interpersonal relationships (EPERIC). Psicothema, 25(2), 275-281. 
Gould, J. (2011). Attachment theory and psychotherapy integration: An introduction and review of the literature. Journal of Psychotherapy Integration, 21(3), 221-231.

Grossmann, K.E., Grossmann, K. y Waters, E. (2005). Attachment from Infancy to Adulthood. New York: Guilford Press.

Hall, D.L., Armstrong, J., Cabral, N. y Kim, Y. (2012). Individual and dyadic effects of adult attachment on quality of life among colorectal cancer care recipients and their caregivers. Psycho-Oncology, 21(supl. I), 67.

Havighurst, R.J. (1972). Developmental task and education. New York: David Mc Kay.

Hernández, M., Cruzado, J.A., Prado, M.C., Rodríguez, E., Hernández, C., González, M.A. y Martín, J.C. (2012). Salud mental y malestar emocional en pacientes con cáncer. Psicooncología, 9(2-3), 299-316.

Hunter, M.J., Davis, P.J. y Tunstall, J.R. (2006). The influence of attachment and emotional support in end-stage cancer. Psycho-Oncology, 15, 431-444.

Huntsinger, E.T. y Luecken, L.J. (2004). Attachment relationships and health behavior: The mediational role of self-esteem. Psychology y Health, 19(4), 515-26.

Kim, Y, Carver, C.S., Deci, E.L. y Kasser, T. (2008). Adult attachment and psychological well-being in cancer caregivers: The mediational role of spouses' motives for caregiving. Health Psychology, 27, 144-154.

Kohn, P.M. y Macdonald, J.E. (1992). The survey of recent life experiences: A decontamitated hassles scale for adults. Journal of Behavioral Medicine, 15(2), 221-236.

Lo, C., Lin, J., Gagliese, L., Zimmermann, C., Mikulincer, M. y Rodin, G. (2010). Age and depression in patients with metastatic cancer: The protective effects of attachment security and spiritual wellbeing. Ageing $y$ Society, 30, 325-336

Lo, C., Walsh, A., Mikulincer, M., Gagliese, L., Zimmermann C. y Rodin, G. (2009). Measuring attachment security in patients with advanced cancer: Psychometric properties of a modified and brief Experiences in Close Relationships scale. Psycho-Oncology, 18, 490-499.

Maunder, R.G. y Hunter, J.J. (2008). Attachment relationships as determinants of physical health. Journal of the American Academy of Psychoanalysis y Dynamic Psychiatry, 36, 11-32.
McWilliams, L.A. y Bailey, S.J. (2010). Associations between adult attachment ratings and health conditions: Evidence from the National Comorbidity Survey Replication. Health Psychology, 29(4), 446-453.

Mikulincer, M. y Shaver, P.R. (2007). Attachment in adulthood: Dynamics, structure, and change. New York: Guilford.

Porter, L.S., Keefe, F.J., Davis, D., Rumble, M. Scipio, C. y Garst, J. (2012). Attachment styles in patients with lung cancer and their spouses: Associations with patient and spouse adjustment. Supportive Care in Cancer, 20(10), 2459-2466.

Sánchez-Cánovas, J. (1994). El bienestar psicológico subjetivo. Escalas de evaluación. Boletín de Psicología, 43, 23-45.

Sandín, B., Chorot, P., Lostao, L., Joiner, T.E., Santed, M.A. y Valiente, R.M. (1999). Escalas PANAS de afecto positivo y negativo: validación factorial y convergencia transcultural. Psicothema, 11(19), 37-51.

Sandín, B., Chorot, P. y Santed, M.A. (1999). Escala SRLE de KohnMacdonald 1992. En B. Sandín (Ed.), El estrés psicosocial: Conceptos y consecuencias clínicas. Madrid: UNED-FUE.

Schmidt, S., Blank, T. Bellizzi, K. y Park, C. (2012). The relationship of coping strategies, social support, and attachment style with posttraumatic growth in cancer survivors. Journal of Health Psychology, 17, 1033-1040.

Snyder, C.F. Garrett-Mayer, E., Brahmer, J.R Carducci, M.A, Pili, R. Stearns, V., Wolff, A.C., Dy, S.M. y Wu, A.W. (2008). Symptoms, supportive care needs, and function in cancer patients: How are they related? Quality of Life Research, 17, 665-677.

Tan, A., Zimmermann, C. y Rodin, G. (2005). Interpersonal processes in palliative care: An attachment perspective on the patient-clinician relationship. Palliative Medicine, 19, 143-150.

Toledo, M., Barreto, M.P., Pascual, A. y Ferrero, J. (1993). Adaptación del Cuestionario de Calidad de Vida de la E.O.R.T.C. para cáncer de mama. Revista de Psicología de la Salud, 5(2), 29-45.

Wallin, D.J. (2007). Attachment in psychotherapy. New York: Guilford Press.

Watson, D., Clarck, L.A. y Tellengen, A. (1998). Development and validation of brief measures of positive and negative affect: The PANAS scales. Journal of Personality and Social Psychology, 54, 1063-1070.

(Artículo recibido: 04-02-2014; revisado: 21-08-2014; aceptado: 05-09-2014) 